\title{
Inter-Relationship between Global Competitiveness and Human Development- Analytical Study of Selected Countries
}

\author{
Mufeed Thinun Younis $^{\mathrm{a}}$ Dina Ahmed Omer ${ }^{\mathrm{b}}$ \\ ${ }^{a}$ College of Administration and Economics, University of Mousl \\ ${ }^{b}$ Technical Institute of Naynawa \\ Omardina004@gmail.com
}

Submission date:- 20/4/2017 Acceptance date:- 7/12/2017 Publication date:- 23/4/2019

Keywords: global competitiveness, Human Development Indicators.

\begin{abstract}
The research aims to study the inter-relationship between global competitiveness indicators and human development indicators for a selected countries (low, middle, and high income)by using Granger Test, and VAR Test. The results of causality test showed that there is a causality relation between the international competitiveness and human development.

We found global competitiveness indicators has clear effect on human development indicators in the low, middle, and high income countries.

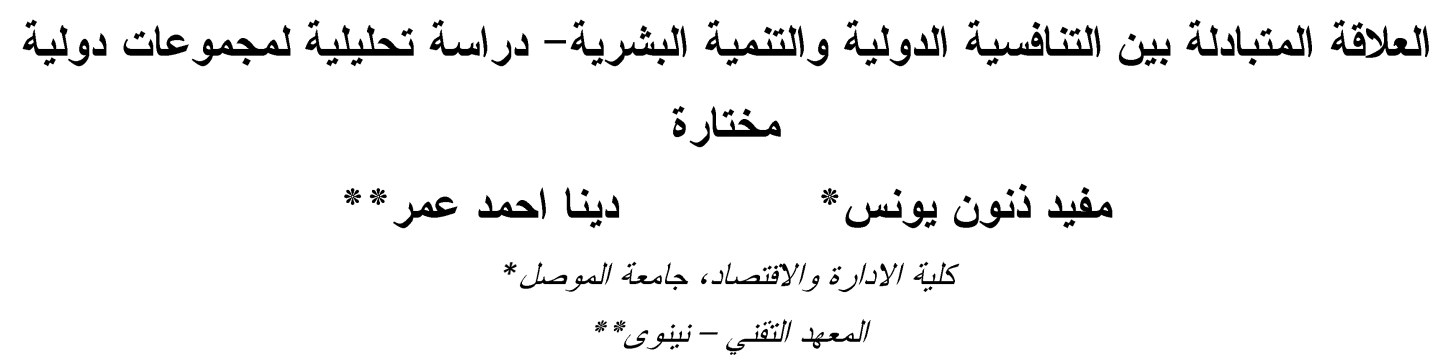

Omardina004@gemail.com
\end{abstract}

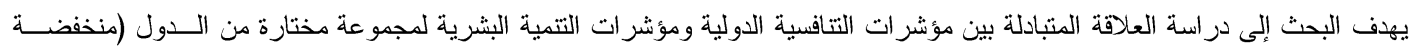

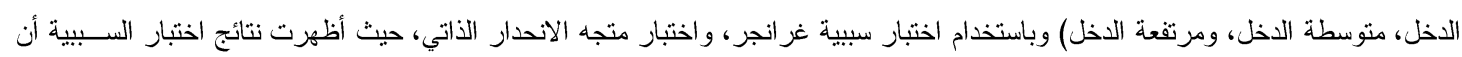

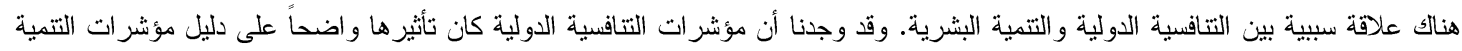
البشرية في الدول المنخفضة، المتوسطة، و المرتفعة الدخل. الكلمات الا الثة: التنافسية الدولية، دليل مؤشر التفات التتمية البشرية.

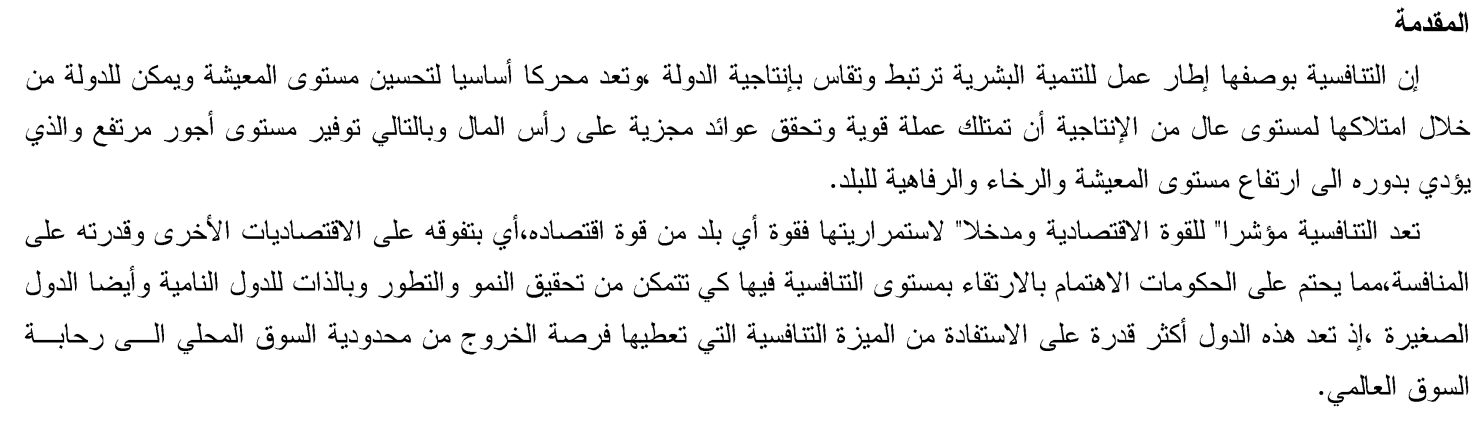


يبدو أن هناك تباينا" في الرؤية (نظريا وتجريبيا) فيما يخص اتجاه العلاقة السبيية بين مؤشرات التتافسية الدولية ومؤشرات التتمية البشرية ضمن المجموعات الدولية ذات مستويات الدخل المختلفة.

هدف البحث

يهدف البحث الى تقدير التأثير المتبادل بين النتافسية الدولية والتتمية البشرية من خلال صياغة أنموذج يقيس العلاقات السبيية بين المتغيرات باستخدام اختبار سبيية غر انجز ومن ثم قياس تلكك العلاقات وتقدير ها وتحليلها لغرض التتبو ء باستخدام أسلوب متجه الانحدار الذاتي.

فرضية البحث

ينطلق البحث من فرضية مفادها أن مؤشرات التتافسية الدولية هي التي تحدد مسارات مؤشرات التتمية البشرية للمجموعات الدولية ذات

المستويات الدخلية المتباينة.

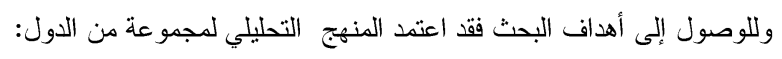

ا. منخفضة الاخل (منغوليا، غينيا، بورندي، هايتي، ليسوتو ، مدغشقر ، بنغلادش، النيبال، كينيا، موريتانيا، أو غندا، نيجيريا، السنغال، موزمبيق،

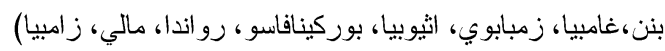

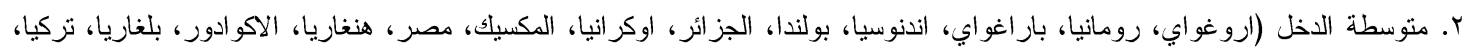

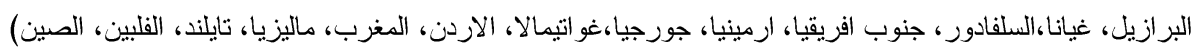

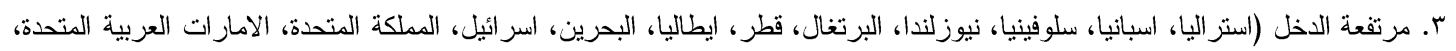

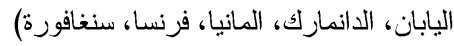

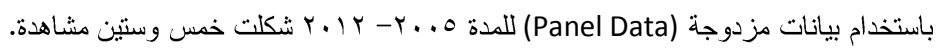

أولا- تأثير التنافسية الدولية في التنمية البشرية

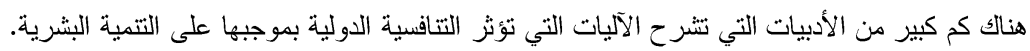

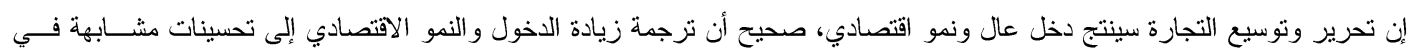

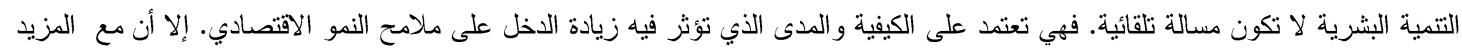

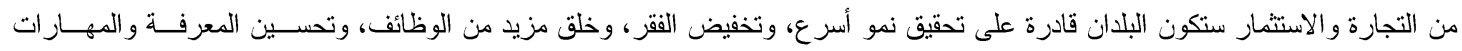

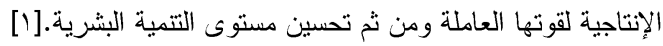
وسع ديفس وكريفان (Davies and Qualivan,2006) بحث العلاقة التقليدية التي تزبط بين الانفتاح التجاري ومتوسط دخـل الفــرد التئـــل التركيز على تأثير التجارة على معايير التتمية الاجتماعية التي تتضمنها معايير التتمية البشرية.

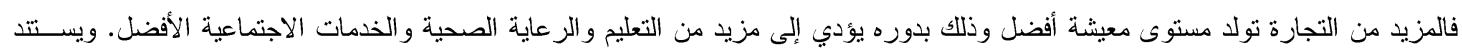

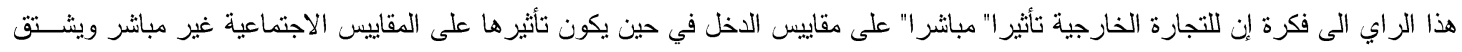

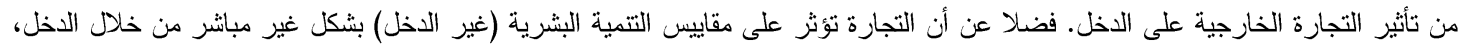

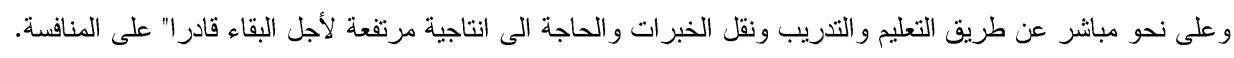

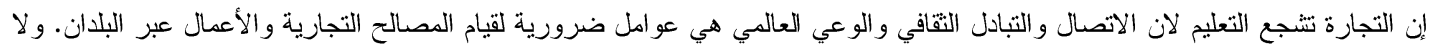

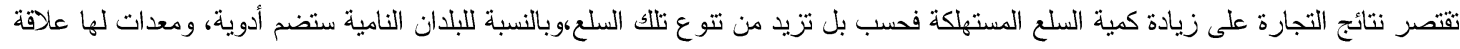

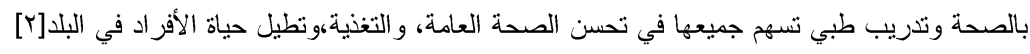

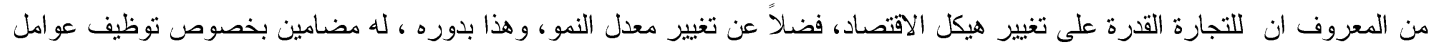
الإنتاج و لاسيما العمل ور أس المال.

ثانيا: تأثير التنمية البشرية في التنافسية الاولية

من جانب أخر فان مستوى التتمية البشرية يؤثر في مستوى التنافسية الدولية.

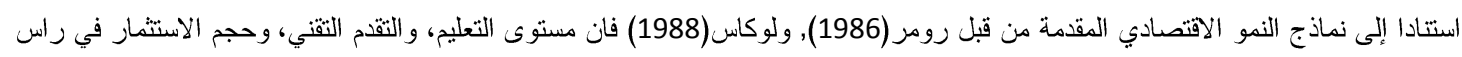

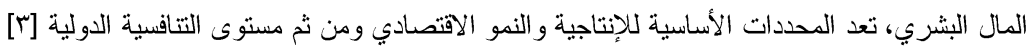
إن الأدلة التجريبية تدعم الافتراض القائل بان مساهمة الأفراد (الأصحاء وجيدي التغذية و المتعلمين) تكون أكبر فـي النــــو الاقتصـادي

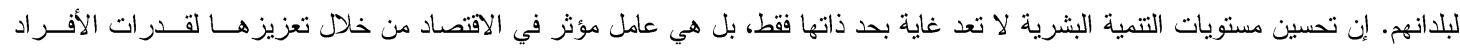


وبالتالي إبداعهم و إنتاجيتهم. وقد أصبح من الواضح أن الصحة والتعليم الأفضل للأفر اد تمثل واحداً من الدحـددات الرئيســة لهيكـل الإنتــاج

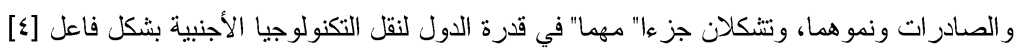

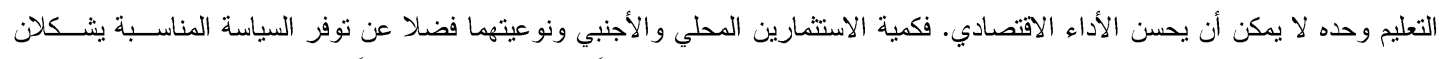

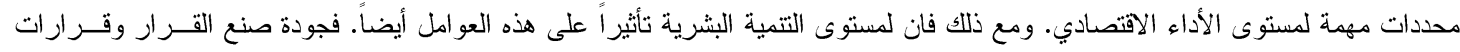

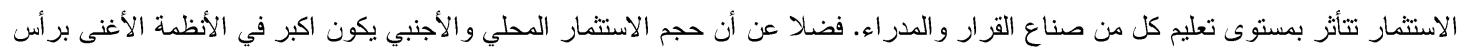

المال البشري [ب]

إن مستوى التعليم الأعلى للقوى العاملة يؤدي الى مستوى أعلى للإنتاجية الكلية لر أس المال لان الأفراد الأكثر تعلماً يكونون أكثــر احتمـالاً

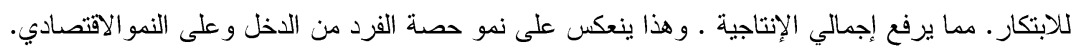

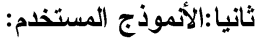

استخدم الأنموذج ذو الصيغة المختزلة (Reduced form econometric model) لتحديد تأثير النتافسية الدولية على التتمية البشرية، ويمكن

كتابة الصيغة الرياضية للأنموذج من خلال الصيغة الاتية:

$H D_{t}=a_{11} H D_{t-1}+a_{12} C_{t-1}+b_{11} H D_{t-2}+b_{12} C_{t-2}+u_{1}+v 1 t$

$C_{t}=a_{21} H D_{t-1}+a_{22} C_{t-1}+b_{21} H D_{t-2}+b_{22} C_{t-2}+u_{2}+v 2 t$

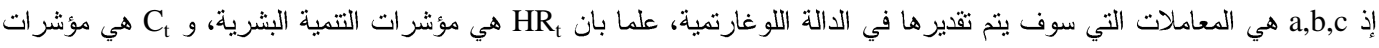
النتافسية الدولية. وبناءا" على ماتقدم فانه سوف يتم اختبار تأثير مؤشرات النتافسية الدولية على مؤشرات التتمية البشرية وذلك بالاختبارات القياسية،التي حددت بفرضية البحث بما يأتي:

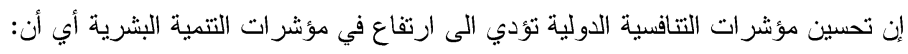
تحسن مؤشرات التتافسية الدولية لايؤدي الى ارتفاع في مؤشرات التتمية البشرية: تحسن مؤشر ات التتافسية الدولية يؤدي الى ارتفاع في مؤشرات التتمية البشرية:

وللتحقق من الفرضية المذكورة أنفا استخدام اختباران،الأول اختبار سبيية الذي يستخدم للتعرف على اتجاه العلاقة السبية والكثف عنها بين

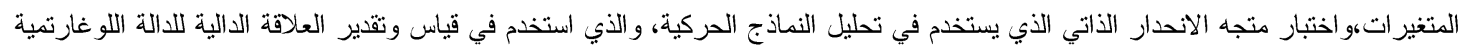
لعينة البلدان المختارة،وقد تضمنت عينة الدول المختارة كل من (دول منخفضة الدخل، دول متوسطة الدخل، دول مرتفعة الدخل) لبيانات مزدوجة r. للفترة 0 (panel Data)

اختبار السببية

يستخدم اختبار السبيية للكثف الإحصائي عن اتجاه العلاقات السبيية(علاقة السبب والتأثير) بين متغيرات الأنموذج، أي أن المتغير المعتمد

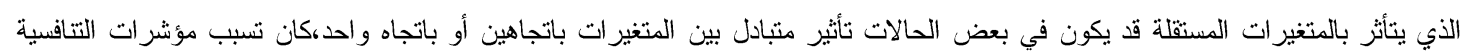

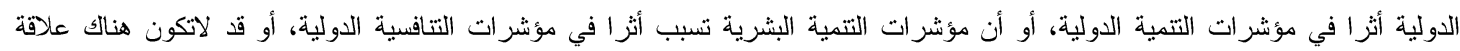

$\mathrm{H}_{\mathrm{o}}: \sum \alpha_{\mathrm{i}}=0$

بينهما.يتطلب اختبار السبيية تحديد فرضية العدم:

$\mathrm{H}_{1}: \sum \alpha_{\mathrm{i}} \neq 0$

ويتم التحقق من الفرضيات السابقة بالاعتماد على اختبار الذي يمكن من خلاله تحديد اتجاه العلاقة السبية بين متغيرات الأنموذج.

متجه الاحدار الذاتي

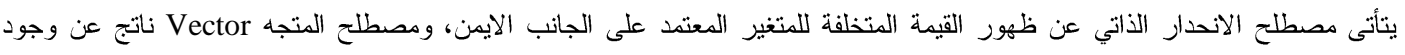

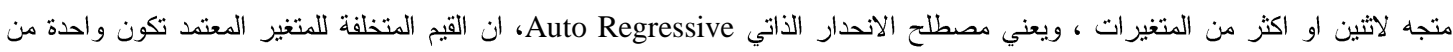
المتغيرات التوضيحية، فاختبار الانحدار الذاتي الذي ياخذ الذئ التخلف الزمني بالحسبان يسمى بنموذج الانحدار الديناميكي، او أنموذج الانحدار

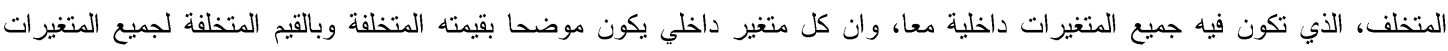
الداخلية في الأنموذج، وقد اثبت هذا النوع من النماذج فائدته في الاقتصاديات النطبيقية، التي تجعل من النظرية الاقتصادية الثابتة نظرية

ديناميكية، من خلال اخذ الزمن بالحسبان. ويستخدم الانحدار الذاتي للمتجه VAR في أنظمة التتبوء للسلاسل الزمنية ذات العلاقة المتبادلة وفي تحليل المعطيات الحركية للتوزيع العشو ائي في متغيرات النظام، والذي يمكن التعبير عنه بالثكل الرياضي لمعادلة الانحدار الاتية: 
2.eg.(5)

$Y_{t}=\mu+\Delta_{1} Y_{t-1}+\Delta P Y_{t-1}+\nu_{t}$

$$
\begin{aligned}
& \text { حيث أن: } \\
& \text { S من المتغيرات الداخلية. }
\end{aligned}
$$

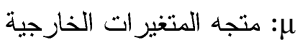

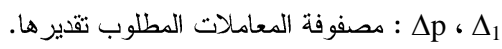

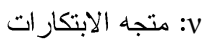

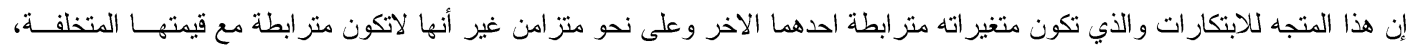

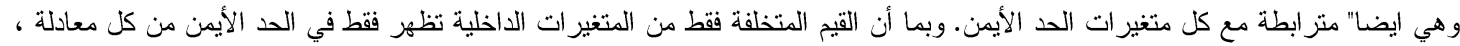

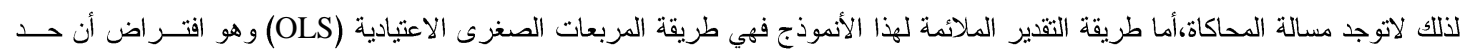

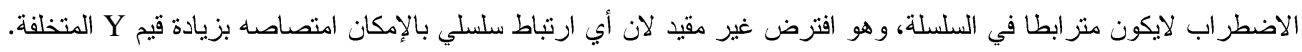

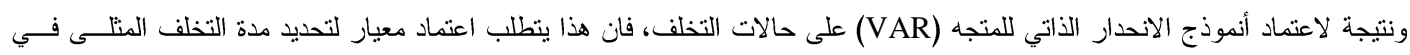

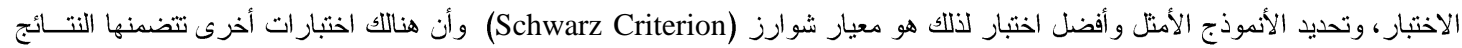

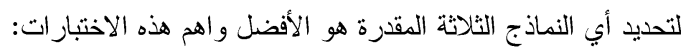
1- اختبار الإمكان الأعظم Likelihood : يعتمد هذا المعيار على تقدير المعلمات المجهولة في احتمالية أن تقدم المشاهدات اكبر قيم للمتغير

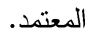
r Akaike Information Criterion:(AIC) يستخدم معيار اكيكي في اختبار النماذج المتتافسة للبدائل غير المستقرة وان القيم

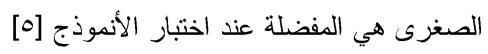
ץ- معيار شوارز Schwarz Criterion : ويستخدم هذا المعيار في تحديد طول مدة التخلف المثلى في أنموذج التخلف-الموزع. ويقترح شوارز $\mathrm{Sc}=\ln \left(\delta^{2}\right)+\operatorname{mln}(\mathrm{n})$

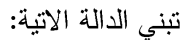

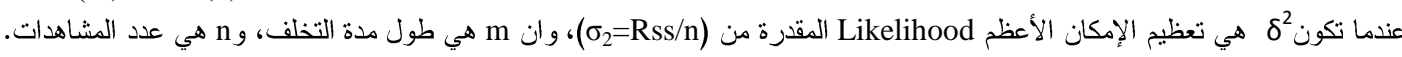

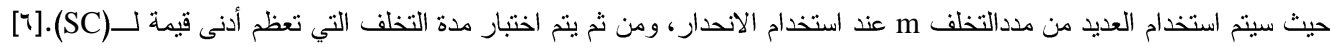
وأن هنالك اختبار ات أخرى تظهر في أنموذج VAR تحدد الأنموذج الأفضل والمعادلة والأفضل منها اختبار الخطــأ المعيـاري ومعامـلـ

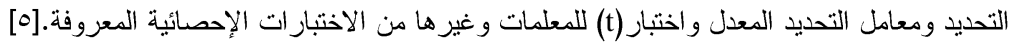

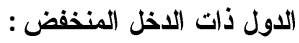
توضح نتائج اختبار السبيية لغر انجر للاول ذات الدخل المنخفض،في الجدول (1) أفضل علاقات سبيية يمكن أن تتحقق في التباطؤ الزمنـي الثاني. وبمقارنة F المحتسبة مع نظيرتها الجدولية تبين ان هناك سبيية باتجاه واحد من المتطلبات الاساسية إلى دليل مؤشرات التتمية البشرية. أي أن

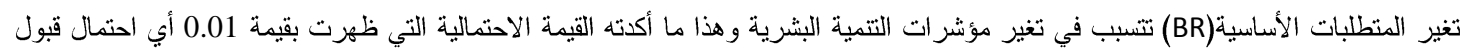
فرضية العدم 0.01. و أظهرت نتائج الاختبار ايضـا" وجود سبية باتجاه واحد من دليل مؤشرات التتمية البشرية باتجاه محفز ات الكفــاءة(EE) أي أن تغيــر فــي مؤشر ات التتمية البشرية يتسبب في تغير محفزات الكفاءة.

\begin{tabular}{|c|c|c|c|}
\hline الاحتمالية & قيمة F المحتسبة & \multicolumn{2}{|c|}{ اتجاه السببية } \\
\hline $\begin{array}{l}0.015 \\
0.009\end{array}$ & $\begin{array}{l}4.329 \\
4.847\end{array}$ & $\mathrm{HDI}$ & $\begin{array}{r}\leftarrow \quad \mathrm{BR} \\
\mathrm{HDI} \leftarrow \mathrm{EE}\end{array}$ \\
\hline
\end{tabular}

جدول (1) اختبار سبيية غرانجر للاول ذات الاخل المنخفض

ننتقل إلى تحليل نتائج السبيية للانحدار الذاني للمتجه (VAR) لهذه المجموعة من الدول ذات الدخل المنخفض، حيث يوضح هذا التحليل تفسير التأثيرات الكمية بين المتغيرات، و الجدول (2) يوضح نتائج اختبار (VAR). 
جدول (2) نتائج أنموذج الاحدار الذاتي للمتجه (VAR) للاول ذات الاخل المنخفض

Vector Autoregression Estimates

Date: 06/05/14 Time: 20:11

Sample (adjusted): 20052012

Included observations: 154 after adjustments

Standard errors in ( ) \& t-statistics in [ ]

\begin{tabular}{|c|c|c|c|c|}
\hline & HDI & $\mathrm{BR}$ & $\mathrm{EE}$ & IF \\
\hline HDI $(-1)$ & $\begin{array}{r}0.693757 \\
(0.05413) \\
{[12.8161]}\end{array}$ & $\begin{array}{r}-0.165070 \\
(0.14401) \\
{[-1.14625]}\end{array}$ & $\begin{array}{c}0.637881 \\
(0.31033) \\
{[2.05547]}\end{array}$ & $\begin{array}{r}0.058597 \\
(0.29003) \\
{[0.20204]}\end{array}$ \\
\hline $\mathrm{BR}(-1)$ & $\begin{array}{r}0.029797 \\
(0.01786) \\
{[1.66827]}\end{array}$ & $\begin{array}{c}0.862935 \\
(0.04752) \\
{[18.1604]}\end{array}$ & $\begin{array}{r}-0.082070 \\
(0.10240) \\
{[-0.80148]}\end{array}$ & $\begin{array}{r}0.198598 \\
(0.09570) \\
{[2.07524]}\end{array}$ \\
\hline $\mathrm{EE}(-1)$ & $\begin{array}{r}0.012972 \\
(0.01185) \\
{[1.09473]}\end{array}$ & $\begin{array}{c}0.071544 \\
(0.03152) \\
{[2.26956]}\end{array}$ & $\begin{array}{r}0.561456 \\
(0.06793) \\
{[8.26506]}\end{array}$ & $\begin{array}{r}0.186298 \\
(0.06349) \\
{[2.93441]}\end{array}$ \\
\hline $\operatorname{IF}(-1)$ & $\begin{array}{r}0.001157 \\
(0.01239) \\
{[0.09332]}\end{array}$ & $\begin{array}{r}0.001200 \\
(0.03297) \\
{[0.03639]}\end{array}$ & $\begin{array}{c}0.122074 \\
(0.07105) \\
{[1.71811]}\end{array}$ & $\begin{array}{r}0.532053 \\
(0.06640) \\
{[8.01250]}\end{array}$ \\
\hline $\mathrm{C}$ & $\begin{array}{r}-0.029145 \\
(0.06575) \\
{[-0.44330]}\end{array}$ & $\begin{array}{r}0.341526 \\
(0.17491) \\
{[1.95260]}\end{array}$ & $\begin{array}{c}1.055215 \\
(0.37692) \\
{[2.79958]}\end{array}$ & $\begin{array}{r}0.077390 \\
(0.35226) \\
{[0.21969]}\end{array}$ \\
\hline R-squared & 0.583165 & 0.732592 & 0.393571 & 0.445520 \\
\hline Adj. R-squared & 0.571974 & 0.725413 & 0.377291 & 0.430635 \\
\hline Sum sq. resids & 0.525387 & 3.718421 & 17.26774 & 15.08234 \\
\hline S.E. equation & 0.059381 & 0.157974 & 0.340428 & 0.318157 \\
\hline F-statistic & 52.11382 & 102.0502 & 24.17515 & 29.93012 \\
\hline Log likelihood & 218.8875 & 68.20479 & -50.03188 & -39.61256 \\
\hline Akaike AIC & -2.777760 & -0.820841 & 0.714700 & 0.579384 \\
\hline Schwarz SC & -2.679158 & -0.722239 & 0.813302 & 0.677986 \\
\hline Mean dependent & 0.421279 & 3.628052 & 3.243896 & 3.068182 \\
\hline S.D. dependent & 0.090764 & 0.301472 & 0.431402 & 0.421644 \\
\hline \multicolumn{2}{|c|}{ Determinant resid covariance (dof adj.) } & $9.26 \mathrm{E}-07$ & & \\
\hline \multicolumn{2}{|c|}{ Determinant resid covariance } & $8.11 \mathrm{E}-07$ & & \\
\hline \multicolumn{2}{|c|}{ Log likelihood } & 205.8264 & & \\
\hline \multicolumn{2}{|c|}{ Akaike information criterion } & -2.413329 & & \\
\hline \multicolumn{2}{|c|}{ Schwarz criterion } & -2.018920 & & \\
\hline
\end{tabular}

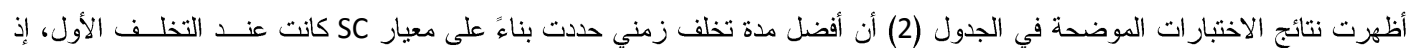

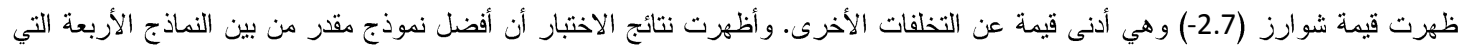

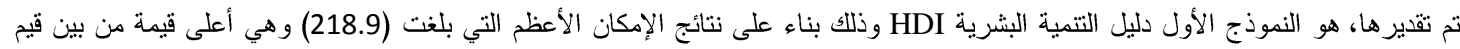

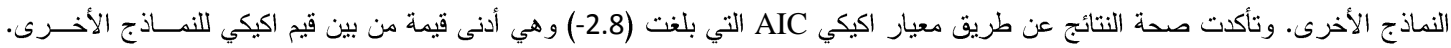

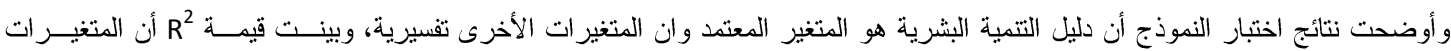
المستقلة تفسر 68\% من التغيرات الحاصلة في المتغير المعتمد.

وتظهر معادلة المنطلبات الأساسية ثأثيرا" معنويا" موجبا" على دليل مؤشرات التتمية البشرية عند التخلف الأول أي أن تغير المتطلبات الأساسية بنسبة 1\% يؤدي الى تغير في دليل التتمية البشرية بنسب 0.03\% في الآجل القصير. 
من جانب أخر ظهز أن لمؤشرات التتمية البشرية تأثيرا" معنويا" موجبا" على محفزات وأن أي تغير في دليل مؤشرات النتمية البشرية بنسبة 1 سيؤدي الى تغير في محفزات الكفاءة بنسبة 0.63\% في الأجل القصير.

وظهر في هذه الدول تأثير متبادل بين دليل مؤشرات التتمية البشرية ومؤشرات التتافسية الدولية (المتطلبات الأساسية،محفز ات الكفاءة) في هـــــ

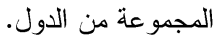

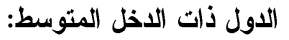

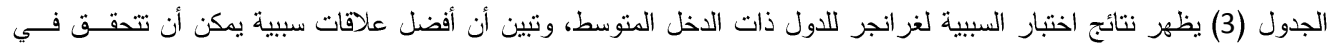

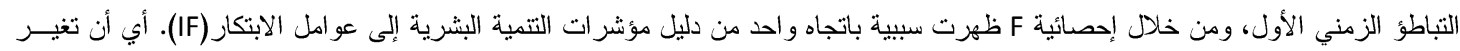

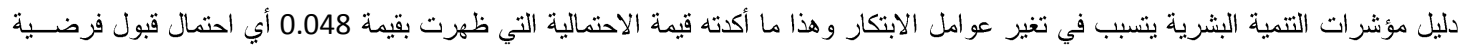

جدول (3) اختبار سببية غرانجر للاول ذات الاخل المتوسط

\begin{tabular}{|c|c|c|}
\hline الاحتمالية & قيمة F (لمحتسبة & اتجاه السبيية \\
\hline 0.048 & 3.957 & $\mathrm{IF} \quad \leftarrow \quad \mathrm{HDI}$ \\
\hline
\end{tabular}

ننتقل إلى اختبارات أنموذج الانحدار الذاتي التي يوضحها الجدول (4)

جدول (4) نتائج أنموذج الاحدار الذاتي للمتجه (VAR) للاول ذات الاخل المتوسط

Vector Autoregression Estimates

Date: 06/05/14 Time: 20:32

Sample (adjusted): 20072012

Included observations: 156 after adjustments

Standard errors in ( ) \& t-statistics in [ ]

\begin{tabular}{|c|c|c|c|c|}
\hline & HDI & BR & EE & IF \\
\hline HDI(-1) & $\begin{array}{r}0.387169 \\
(0.08473) \\
{[4.56960]}\end{array}$ & $\begin{array}{r}-\mathbf{0 . 0 5 5 2 8 2} \\
(0.33124) \\
{[-0.16689]}\end{array}$ & $\begin{array}{r}0.492734 \\
(0.43089) \\
{[1.14353]}\end{array}$ & $\begin{array}{r}-0.064734 \\
(0.24468) \\
{[-0.26457]}\end{array}$ \\
\hline HDI(-2) & 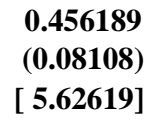 & $\begin{array}{r}-0.229126 \\
(0.31699) \\
{[-0.72281]}\end{array}$ & $\begin{array}{c}\mathbf{0 . 3 4 0 5 4 1} \\
(0.41236) \\
{\left[\begin{array}{l}0.82584 \\
\end{array}\right.}\end{array}$ & $\begin{array}{c}-0.101474 \\
(0.23416) \\
{[-0.43336]}\end{array}$ \\
\hline BR(-1) & $\begin{array}{r}-0.013782 \\
(0.02434) \\
{[-0.56618]}\end{array}$ & $\begin{array}{r}\mathbf{0 . 2 7 9 8 9 9} \\
(0.09517) \\
{[2.94108]}\end{array}$ & $\begin{array}{r}0.295369 \\
(0.12380) \\
{[2.38586]}\end{array}$ & $\begin{array}{r}\mathbf{0 . 0 2 8 8 9 2} \\
(\mathbf{0 . 0 7 0 3 0}) \\
{\left[\begin{array}{l}0.41099 \\
\end{array}\right.}\end{array}$ \\
\hline BR(-2) & $\begin{array}{r}\mathbf{0 . 0 2 0 8 1 4} \\
(\mathbf{0 . 0 2 7 0 8}) \\
{\left[\begin{array}{l}0.76867]\end{array}\right.}\end{array}$ & 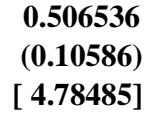 & $\begin{array}{r}-0.250352 \\
(0.13771) \\
{[-1.81796]}\end{array}$ & $\begin{array}{r}\mathbf{0 . 0 3 8 6 0 8} \\
(0.07820) \\
{\left[\begin{array}{l}0.49372 \\
\end{array}\right.}\end{array}$ \\
\hline EE(-1) & $\begin{array}{c}-0.042021 \\
(0.02848) \\
{[-1.47560]}\end{array}$ & $\begin{array}{r}\mathbf{0 . 1 7 9 4 0 1} \\
(\mathbf{0 . 1 1 1 3 3}) \\
{\left[\begin{array}{l}1.61142]\end{array}\right.}\end{array}$ & $\begin{array}{r}\mathbf{0 . 4 3 1 4 9 9} \\
(0.14482) \\
{\left[\begin{array}{l}2.97947]\end{array}\right.}\end{array}$ & 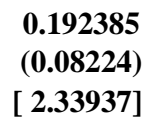 \\
\hline EE(-2) & $\begin{array}{r}\mathbf{0 . 0 3 7 0 7 4} \\
(\mathbf{0 . 0 2 9 2 7}) \\
{\left[\begin{array}{l}1.26678]\end{array}\right.}\end{array}$ & 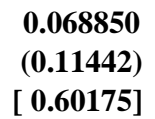 & $\begin{array}{r}\mathbf{0 . 1 8 5 3 2 9} \\
(\mathbf{0 . 1 4 8 8 4}) \\
{\left[\begin{array}{l}1.24518 \\
\end{array}\right.}\end{array}$ & $\begin{array}{r}-0.041086 \\
(0.08452) \\
{[-0.48613]}\end{array}$ \\
\hline IF $(-1)$ & $\begin{array}{r}6.71 E-05 \\
(0.01896) \\
{[0.00354]}\end{array}$ & $\begin{array}{r}\mathbf{- 0 . 0 7 2 4 2 7} \\
(\mathbf{0 . 0 7 4 1 2}) \\
{[-0.97711]}\end{array}$ & $\begin{array}{c}\mathbf{0 . 0 5 4 9 0 4} \\
(\mathbf{0 . 0 9 6 4 2 )} \\
{[\mathbf{0 . 5 6 9 4 0}]}\end{array}$ & $\begin{array}{r}\mathbf{0 . 8 2 1 9 4 5} \\
(\mathbf{0 . 0 5 4 7 5 )} \\
{[15.0116]}\end{array}$ \\
\hline IF $(-2)$ & -0.002450 & $\mathbf{0 . 0 7 7 8 1 5}$ & 0.019325 & -0.063723 \\
\hline
\end{tabular}




\begin{tabular}{|c|c|c|c|c|}
\hline \multirow[b]{2}{*}{$\mathbf{C}$} & $\begin{array}{c}(0.01531) \\
{[-0.16002]}\end{array}$ & $\begin{array}{r}(0.05987) \\
{[1.29984]}\end{array}$ & 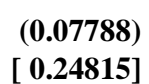 & $\begin{array}{r}(0.04422) \\
{[-1.44098]}\end{array}$ \\
\hline & 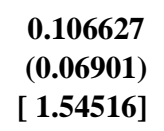 & $\begin{array}{r}\mathbf{0 . 1 3 9 7 5 2} \\
(\mathbf{0 . 2 6 9 7 8}) \\
{\left[\begin{array}{l}0.51802 \\
\end{array}\right.}\end{array}$ & $\begin{array}{c}\mathbf{0 . 4 9 8 5 5 4} \\
(\mathbf{0 . 3 5 0 9 4 )} \\
{\left[\begin{array}{l}1.42061]\end{array}\right.}\end{array}$ & $\begin{array}{r}\mathbf{0 . 0 6 5 6 4 6} \\
(0.19928) \\
{\left[\begin{array}{l}0.32941]\end{array}\right.}\end{array}$ \\
\hline R-squared & 0.512939 & 0.722941 & 0.569254 & 0.863090 \\
\hline Adj. R-squared & 0.486432 & 0.707863 & 0.545812 & 0.855639 \\
\hline Sum sq. resids & 0.533934 & 8.160685 & 13.80947 & 4.452887 \\
\hline S.E. equation & 0.060268 & 0.235616 & 0.306500 & 0.174045 \\
\hline F-statistic & 19.35125 & 47.94664 & 24.28352 & 115.8374 \\
\hline Log likelihood & 221.4780 & 8.786769 & -32.24331 & 56.03725 \\
\hline Akaike AIC & -2.724076 & 0.002734 & 0.528760 & -0.603042 \\
\hline Schwarz SC & -2.548123 & 0.178687 & 0.704714 & -0.427088 \\
\hline Mean dependent & 0.709705 & 4.389808 & 4.004231 & 3.550705 \\
\hline S.D. dependent & 0.084098 & 0.435925 & 0.454791 & 0.458076 \\
\hline \multicolumn{2}{|c|}{ Determinant resid covariance (dof adj.) } & 5.60E-07 & & \\
\hline \multicolumn{2}{|c|}{ Determinant resid covariance } & 4.41E-07 & & \\
\hline \multicolumn{2}{|c|}{ Log likelihood } & 256.0252 & & \\
\hline \multicolumn{2}{|c|}{ Akaike information criterion } & -2.820836 & & \\
\hline \multicolumn{2}{|c|}{ Schwarz criterion } & -2.117023 & & \\
\hline
\end{tabular}

تبين أن أفضل النماذج هو الأنموذج الذي يستخدم دليل التتمية البشرية بناء على نتائج التي بلغت (221.5) وهي أعلى قيمة مـن بـين قـيم

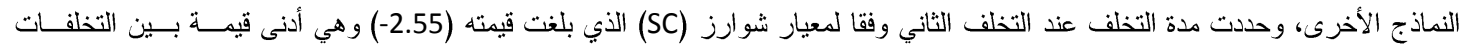

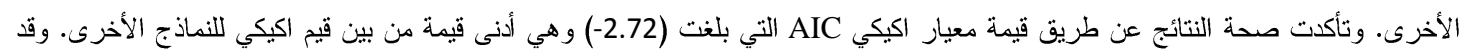

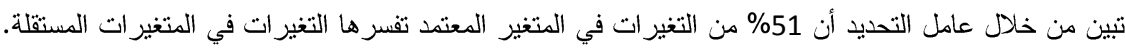

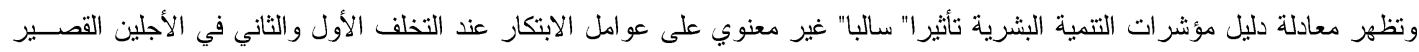
و الطويل. وبالرغم من عدم ظهور تأثير معنوي للمتغيرات التفسيرية إلا انه قد تأثنر لدينا أن ( BR و EE) تأثير ا" موجبا" عند التخلف الثاني. أماFل فكـان تأثير ها سالبا عند التخلف الثاني.

و هذه النتائج تطايق تقريبا مع نتائج سبيية غرانجر، حيث يظهر في هذه المجموعة من الدول ذات الدخل المتوسط أن دليل مؤشرات النتمية البشرية يؤثر في متغيرات التنافسية الدولية (عوامل الابتكار).

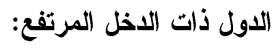
يوضح الجدول (5) سبيية غرانجر في الدول ذات الدخل المرتفع عند التخلف الأول. حيث أظهرت النتائج أن هناك سبيية باتجاه واحد من محفزات الكفاءة إلى دليل مؤشرات التتمية البشرية وقد بلغت قيمة F المحتسبة 3.918 وهي أكبر من الجدولية عند احتمالية 0.050 باستخدام تخلف لسنة واحدة. كما أن هناك سبيية باتجاه واحد من عو امل الابتكار باتجاه دليل مؤشرات التتمية البشرية بدلالة قيمة F المحتسبة 5.738 وهي اكبر من الجدوليــة

جدول (5) اختبار سبيية غر اتجر للاول ذات الاخل المرتفع

\begin{tabular}{|c|c|c|}
\hline الاحتمالية & قيمة F المحتسبة & اتجاه السبيية \\
\hline $\begin{array}{l}0.050 \\
0.018\end{array}$ & $\begin{array}{l}3.918 \\
5.738\end{array}$ & $\begin{array}{l}\mathrm{EE} \leftarrow \mathrm{HDI} \\
\text { HDI } \leftarrow \text { IF }\end{array}$ \\
\hline
\end{tabular}

أما نتائج اختبار ات الانحدار الذاتي للمتجه (VAR) للاول ذات الاخل المرتفع فنظهر في الجدول (6). 
Vector Autoregression Estimates

Date: 06/05/14 Time: 20:35

Sample (adjusted): 20072012

Included observations: 102 after adjustments

Standard errors in ( ) \& t-statistics in [ ]

\begin{tabular}{|c|c|c|c|c|}
\hline & HDI & BR & EE & IF \\
\hline HDI(-1) & $\begin{array}{r}\mathbf{0 . 0 2 0 3 5 8} \\
(\mathbf{0 . 0 9 0 0 6 )} \\
{\left[\begin{array}{l}0.22605]\end{array}\right.}\end{array}$ & $\begin{array}{r}-0.078666 \\
(0.44084) \\
{[-0.17845]}\end{array}$ & $\begin{array}{r}\mathbf{0 . 0 9 9 1 4 8} \\
(\mathbf{0 . 2 5 3 8 5}) \\
{\left[\begin{array}{l}0.39058 \\
\end{array}\right.}\end{array}$ & 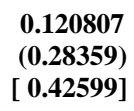 \\
\hline HDI(-2) & $\begin{array}{r}\mathbf{0 . 5 0 3 3 4 8} \\
\mathbf{( 0 . 0 9 3 3 2 )} \\
{\left[\begin{array}{r}5.39401]\end{array}\right.}\end{array}$ & $\begin{array}{r}\mathbf{0 . 0 9 7 7 0 3} \\
(0.45678) \\
{\left[\begin{array}{l}0.21390]\end{array}\right.}\end{array}$ & $\begin{array}{r}0.391315 \\
(0.26302) \\
{\left[\begin{array}{l}1.48775 \\
\end{array}\right.}\end{array}$ & $\begin{array}{r}\mathbf{0 . 0 7 9 2 6 8} \\
(0.29384) \\
{\left[\begin{array}{l}0.26976 \\
\end{array}\right.}\end{array}$ \\
\hline BR(-1) & $\begin{array}{r}\mathbf{0 . 0 0 0 8 3 2} \\
(\mathbf{0 . 0 2 0 3 8}) \\
{\left[\begin{array}{l}0.04083 \\
\end{array}\right.}\end{array}$ & 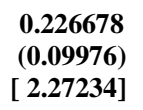 & $\begin{array}{r}\mathbf{0 . 0 9 7 3 0 1} \\
(0.05744) \\
{\left[\begin{array}{l}1.69390 \\
\end{array}\right.}\end{array}$ & $\begin{array}{r}\mathbf{0 . 0 9 7 2 9 9} \\
(0.06417) \\
{\left[\begin{array}{l}1.51620 \\
\end{array}\right.}\end{array}$ \\
\hline BR(-2) & $\begin{array}{r}\mathbf{0 . 0 3 4 7 4 9} \\
(0.02840) \\
{[1.22357]}\end{array}$ & 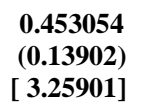 & $\begin{array}{c}\mathbf{0 . 1 8 5 9 3 8} \\
(\mathbf{0 . 0 8 0 0 5}) \\
{\left[\begin{array}{l}2.32279 \\
\end{array}\right.}\end{array}$ & $\begin{array}{r}-\mathbf{0 . 1 3 0 6 5 2} \\
(0.08943) \\
{[-1.46096]}\end{array}$ \\
\hline EE(-1) & $\begin{array}{r}-0.006078 \\
(0.02777) \\
{[-0.21884]}\end{array}$ & 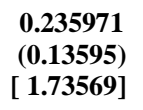 & $\begin{array}{r}\mathbf{0 . 1 5 8 0 3 5} \\
(\mathbf{0 . 0 7 8 2 9}) \\
{\left[\begin{array}{l}2.01870 \\
\end{array}\right.}\end{array}$ & $\begin{array}{r}\mathbf{- 0 . 1 4 2 1 9 0} \\
\mathbf{( 0 . 0 8 7 4 6 )} \\
{[-1.62580]}\end{array}$ \\
\hline EE(-2) & $\begin{array}{r}-\mathbf{0 . 0 1 5 3 4 4} \\
(0.02980) \\
{[-0.51499]}\end{array}$ & 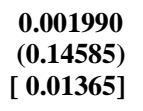 & $\begin{array}{r}\mathbf{0 . 3 0 8 5 3 2} \\
(\mathbf{0 . 0 8 3 9 8}) \\
{\left[\begin{array}{l}3.67376 \\
\end{array}\right.}\end{array}$ & $\begin{array}{c}\mathbf{0 . 3 3 2 3 5 2} \\
(\mathbf{0 . 0 9 3 8 2}) \\
{\left[\begin{array}{l}3.54233 \\
\end{array}\right.}\end{array}$ \\
\hline IF $(-1)$ & $\begin{array}{r}\mathbf{0 . 0 3 8 9 9 9} \\
(\mathbf{0 . 0 2 1 8 9}) \\
{\left[\begin{array}{l}1.78144]\end{array}\right.}\end{array}$ & $\begin{array}{r}-0.023991 \\
(0.10716) \\
{[-0.22389]}\end{array}$ & 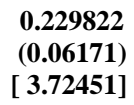 & 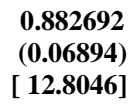 \\
\hline IF $(-2)$ & $\begin{array}{r}\mathbf{- 0 . 0 3 1 9 1 2} \\
\mathbf{( 0 . 0 2 2 3 8 )} \\
{[-1.42576]}\end{array}$ & $\begin{array}{r}-0.017327 \\
(0.10956) \\
{[-0.15815]}\end{array}$ & $\begin{array}{r}-\mathbf{- 0 . 1 3 7 1 3 8} \\
(0.06309) \\
{[-2.17373]}\end{array}$ & $\begin{array}{r}-\mathbf{- 0 . 1 1 1 1 0 8} \\
(0.07048) \\
{[-1.57642]}\end{array}$ \\
\hline $\mathbf{C}$ & 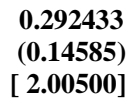 & 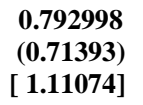 & $\begin{array}{r}0.213446 \\
(0.41110) \\
{\left[\begin{array}{l}0.51920 \\
\end{array}\right.}\end{array}$ & $\begin{array}{r}\mathbf{0 . 1 4 9 5 7 3} \\
(\mathbf{0 . 4 5 9 2 7}) \\
{[\mathbf{0 . 3 2 5 6 7}]}\end{array}$ \\
\hline $\begin{array}{l}\text { R-squared } \\
\text { Adj. R-squared } \\
\text { Sum sq. resids } \\
\text { S.E. equation } \\
\text { F-statistic } \\
\text { Log likelihood } \\
\text { Akaike AIC } \\
\text { Schwarz SC } \\
\text { Mean dependent } \\
\text { S.D. dependent }\end{array}$ & \begin{tabular}{r|}
0.301729 \\
0.241663 \\
0.692112 \\
0.086267 \\
5.023274 \\
109.9103 \\
-1.978633 \\
-1.747018 \\
0.879382 \\
0.099064
\end{tabular} & $\begin{array}{r}0.409137 \\
0.358310 \\
16.58320 \\
0.422272 \\
8.049609 \\
\mathbf{5 2 . 0 8 6 0 2} \\
1.197765 \\
1.429380 \\
5.496667 \\
0.527145\end{array}$ & $\begin{array}{l}0.705607 \\
0.680283 \\
5.498653 \\
0.243157 \\
27.86306 \\
4.212221 \\
0.093878 \\
0.325493 \\
4.904902 \\
0.430035\end{array}$ & $\begin{array}{r}0.854260 \\
0.841723 \\
6.862728 \\
0.271648 \\
68.14023 \\
-7.089475 \\
0.315480 \\
0.547095 \\
4.639608 \\
0.682807\end{array}$ \\
\hline $\begin{array}{l}\text { Determinant resid c } \\
\text { Determinant resid c } \\
\text { Log likelihood } \\
\text { Akaike information } \\
\text { Schwarz criterion }\end{array}$ & adj.) & $\begin{array}{r}5.30 \mathrm{E}-06 \\
3.66 \mathrm{E}-06 \\
\mathbf{5 9 . 4 6 9 8 2} \\
-\mathbf{0 . 4 6 0 1 9 2} \\
0.466269\end{array}$ & & \\
\hline
\end{tabular}

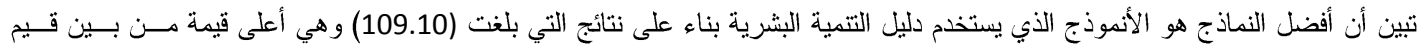

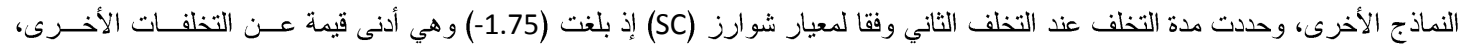

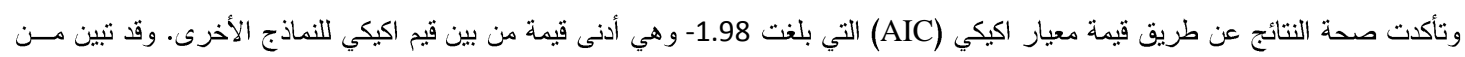

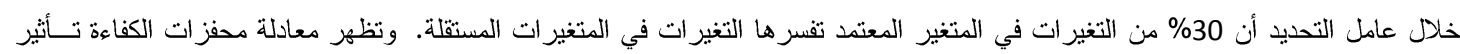
غير معنوي على دليل مؤشر ات التتمية البشرية عند التخلف الأول و الثاني. 


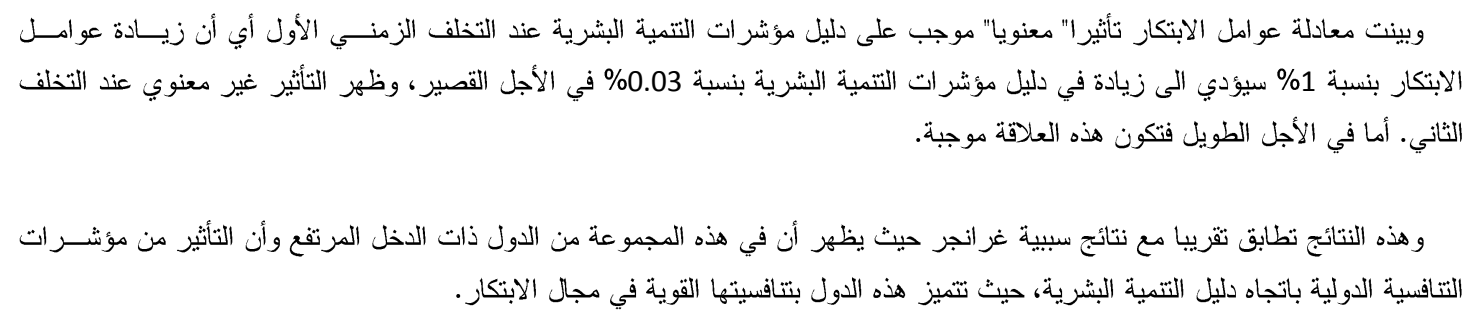

[1] A.Amin aA,Hamdi.,"Global competitiveness and economic growth: empirical verification for African countries".(2012).

www.ccsenet.org/journal/index.php/ijef/article/.../11565/

[2] A.Davies \& G.Quinliva,"A Panel Data Analysis of the Impact of Trade on Human Development".(2006) www.antolin-davies.com/research/hdi.pdf.

[3] G.Ranis and F. Stewart,"Dynamic links between the economy and development".(2005).

www.un.org/esa/desa/papers/2005/wp8_2005.pdf

[4] A.Ramirez and G, Ranis and F, Stewart,"Economic growth and human development".(1991).

www.econ.yale.edu/gowth-pdf/cdp787.pdf.

[5] W.Greene."Econometric Analysis", Fourth Edition, prentice hall, New Jersey,2002.

[6] D.Gujarati, " Basic Econometrics", Third Edition, McGraw-Hill, Singapore, 1995. 